\title{
THE GAUSSIAN APPROXIMATION FOR A PURELY INDUCTIVE WAKE FUNCTION
}

\author{
S. Petracca, Th. Demma, University of Sannio, Benevento, Italy and INFN, \\ K. Hirata, Sokendai, The Graduate University for Advanced Studies, Hayama, Japan
}

\begin{abstract}
The equilibrium bunch length in electron storage rings with a purely inductive localized wake function has been studied, assuming the Gaussian approximation of the distribution function in phase space. The localized wake describes more general cases, even uniformly distributed wakes, described by the Haissinski equation. The comparison of our results with those obtained from Haissinski equation shows good agreement and helps understanding the validity of the Gaussian approximation.
\end{abstract}

\section{INTRODUCTION}

The wake force describes the electromagnetic interaction between particles and environment and affects the particles distribution in a bunch. In the conventional analytic approach one assumes that the source of the wake force is uniformly distributed along the ring, and the equilibrium bunch distribution at low current is a solution of the Haissinski equation, the so-called Potential Well Distortion (PWD) equation. A linear stability analysis around this static solution gives the threshold for turbulent bunch lengthening. Obviously this method tells us very little about the behavior of the unstable solutions. On the other hand it is more realistic for large machines to consider the many sources of wake fields along the ring as localized objects, as usually assumed in the Multi-Particle Tracking (MPT) codes.

In a previous paper [1], the authors studied an analitical model which incorporates the time dependence of the wake force, assuming that if the initial distribution function in synchrotron phase space is Gaussian, it can still be approximated by a Gaussian after the effect of the wake force, represented as a 'kick'. The distribution function can be accordingly always represented by its only first and second order moments, describing the beam envelope in synchrotron phase space. The evolution of these moments from turn to turn can be obtained by applying a non linear mapping at each turn. The equilibrium buch length can have stable solutions with period-one fixed points as well as multistable states or a cusp-catastrophe behavior, depending on the parameters values. The results obtained in [1] show a good agreement with those obtained from MPT.

The localized wake can be extended to more general cases, even to uniformly distributed wakes, described by the Haissinski equation. To do so one should introduce the superperiodicity $N_{s}$ and let it grow to infinity.

In this paper we study the validity of the Gaussian ap- proximation with respect to the existence of the equilibrium longitudinal distribution of electrons in circular accelerators in the case of a purely inductive localized wake function.

\section{THE MOMENT MAPPING}

The longitudinal beam dynamics in electron storage rings can be described by the stochastic equations of motion for a single particle (Langevin equations). Introducing the canonical variables:

$$
\begin{gathered}
x_{1}=\frac{\text { longitudinal displacement }}{\text { natural bunch length }}, \\
x_{2}=\frac{\text { relative energy spread }}{\text { natural energy spread }} .
\end{gathered}
$$

and integrating the Langevin equations over one turn, we obtain the following stochastic mapping:

$$
\left(\begin{array}{l}
x_{1} \\
x_{2}
\end{array}\right)^{\prime}=U\left(\begin{array}{c}
x_{1} \\
\Lambda x_{2}+\hat{r} \sqrt{1-\Lambda^{2}}-\phi\left(x_{1}\right)
\end{array}\right)
$$

where $\vec{X}^{\prime}=\left(x_{1}^{\prime}, x_{2}^{\prime}\right)$ is $\vec{X}=\left(x_{1}, x_{2}\right)$ after one turn. Here $U$ is the rotation matrix:

$$
U=\left(\begin{array}{cc}
\cos \mu & \sin \mu \\
-\sin \mu & \cos \mu
\end{array}\right)
$$

$\mu=2 \pi \nu_{s}, \quad \nu_{s}$ being the synchrotron tune, $\Lambda=$ $\exp (-2 / T), T$ being the synchrotron damping time measured in units of the revolution period, $\hat{r}$ is a Gaussian random variable with $<\hat{r}>=0$ and $<\hat{r}^{2}>=1$. The wake force $\phi\left(x_{1}\right)$ is represented by:

$$
\phi\left(x_{1}\right)=\frac{Q_{t o t}}{\sigma_{0} E_{0}} \int_{0}^{\infty} \rho(x-u) W(u) d u .
$$

where $E_{0}$ is the nominal beam energy, $\sigma_{0}$ is the nominal relative energy spread ( $\sigma_{0} E_{0}$ is the natural energy spread), $W(x)$ is the wake potential and $\rho(x)$ is the charge density normalized to one. Note that synchrotron oscillations have been linearized, and radiation is localized at one point of the ring [3]. The above stochastic mapping is equivalent to an infinite hierarchy of deterministic mappings in the following statistical quantities: $\bar{x}_{i}=\left\langle x_{i}\right\rangle$, $\sigma_{i j}=<\left(x_{i}-\bar{x}_{j}\right)\left(x_{j}-\bar{x}_{j}\right)>$, and so on, which are the moments of the distribution function $\psi(\vec{x}),\langle *\rangle$ indicating an average over all particles. Our main assumption 
is that the distribution function in phase space is always a Gaussian, even in the presence of a wake force:

$$
\psi\left(x_{1}, x_{2}\right)=\frac{\exp \left[\frac{1}{2} \sum_{i, j}^{2} \sigma_{i, j}^{-1}\left(x_{i}-\bar{x}_{i}\right)\left(x_{j}-\bar{x}_{j}\right)\right]}{2 \pi \sqrt{\operatorname{det} \sigma}} .
$$

We consider a purely inductive wake function

$$
W(x)=b \delta^{\prime}(x)
$$

and split the mapping for the second order moments into three parts, representing the effect of radiation, wake-force and synchrotron oscillation, as it follows:

radiation:

$$
\begin{aligned}
\sigma_{11}^{\prime} & =\sigma_{11} \\
\sigma_{12}^{\prime} & =\Lambda \sigma_{12} \\
\sigma_{22}^{\prime} & =\Lambda^{2} \sigma_{22}+\left(1-\Lambda^{2}\right)
\end{aligned}
$$

wake force:

$$
\begin{aligned}
\sigma_{11}^{\prime} & =\sigma_{11} \\
\sigma_{12}^{\prime} & =\sigma_{12}+\frac{b}{4 \sqrt{\pi \sigma_{11}}} \\
\sigma_{22}^{\prime} & =\sigma_{22}+\frac{b \sigma_{12}}{2 \sigma_{11} \sqrt{\pi \sigma_{11}}}+\frac{b^{2}}{6 \sigma_{11}^{2} \pi \sqrt{3}},
\end{aligned}
$$

synchrotron oscillation:

$$
\sigma_{i j}^{\prime}=\sum_{h, k=1}^{2} U_{i h} \sigma_{h k} U_{k j}^{t} .
$$

The stability of the system depends on the values of the synchrotron tune $\nu_{s}$, the damping time (measured in number of turns) $T_{0}$ and the strength of the wake force $b$. We studied a wide range of parameters values and found stable solution of period-one and period-two, multi-stable states and coexistence of solutions with different periodicity. These results are in very good agreement with those obtained in [4], but in addition to them we found fine structures of bifurcations and chaotic regions, depending on parameters values.

As an example we plot $\sigma_{11}$ versus $b$ in Fig. 1 (top) for $T=30$ and $\nu_{s}=0.085$ with $N_{S}=1$. In the parameter space a chaotic region shows up and this behavior mainly depends on the $b$ value but is almost indipendent on $\nu_{s}$ and $T$.

The localized wake can be extended to more general cases, even to uniformly distributed wakes, described by the Haissinski equation. To do so one should introduce the superperiodicity $N_{s}$ and let it grow to infinity. This is done introducing in the mapping the following substitutions:

$$
\nu_{s} \longrightarrow \nu_{s} / N_{s}, \quad T_{0} \longrightarrow T_{0} N_{s}, \quad b \longrightarrow b / N_{s}
$$

In Fig. 1 (bottom) we show $\sigma_{11}(b)$ with $N_{S}=150$ : the chaotic behavior exists also for $N_{s}>>1$. In Fig. 2 we plot $\sigma_{11}$ versus $b$ for different $N_{s}$ and $b>0$. As $N_{s}$ increases the mapping curves become more closely spaced and $\sigma_{11}(b)$ converges to the solution of the PWD equation.
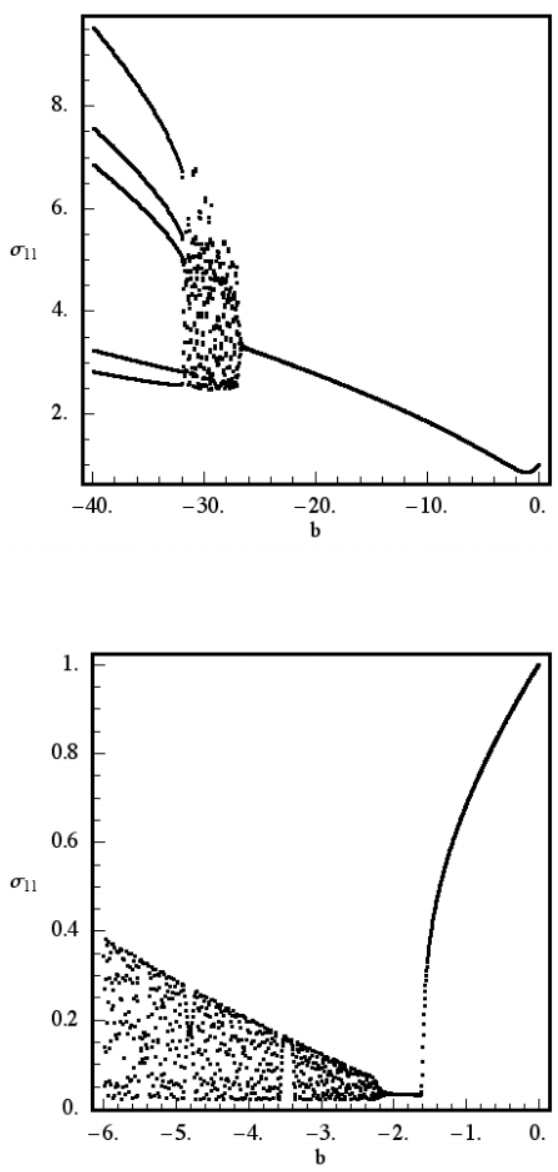

Figure 1: $\sigma_{11}$ versus $b$ for $T=30, \nu_{s}=0.085$ and with (top) $N_{S}=1$ and (bottom) $N_{S}=150$.

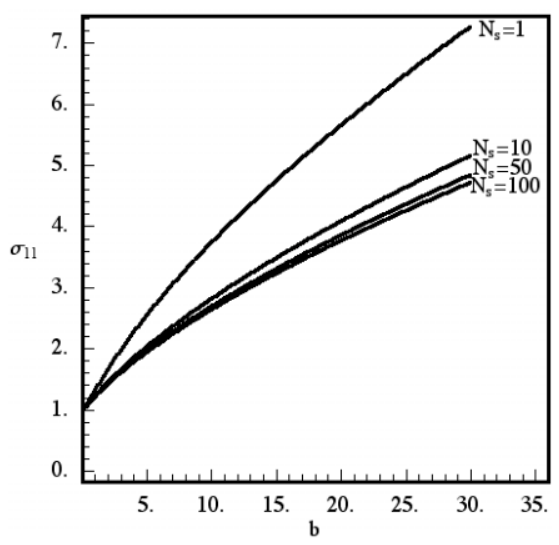

Figure 2: $\sigma_{11}$ versus $b$ for different $N_{s}$ and $T=30, \nu_{s}=$ 0.085 . 


\section{HAISSINSKI EQUATION}

Introducing the variables $x=\omega_{0} x_{1}, y=\omega_{0} x_{2}$ the Haissinski equation can be written as [5]:

$$
u(x)=K \exp \left\{-\frac{x^{2}}{\gamma^{2}}-\lambda_{0} \int_{0}^{+\infty} S(y) u(x-y) d y\right\}
$$

with the normalization condition

$$
\int_{-\infty}^{+\infty} u(x) d x=1
$$

and the following parameter definitions: $\omega_{0}=2 \pi / T_{0}, \gamma=$ $\sqrt{2} \omega_{0} \sigma, \lambda_{0}=2 N \omega_{0} V_{R F}(0) / \dot{V}_{R F}(0) \gamma^{2}$,

$$
S(y)=\frac{e \omega_{0}}{V_{R F}(0)} \int_{0}^{y} W(\xi) d \xi
$$

where $N$ is the total bunch particles and $\sigma$ the bunch length. Following [2] we rewrite equation (4) and (9) as

$$
\log u(x)+\lambda u(x)=\log K-\frac{x^{2}}{\gamma^{2}},
$$

with $\lambda=e N b / \dot{V}_{R F}(0) \sigma^{2}$. For $\lambda \geq 0$ the solution exists always, but not for $\lambda \leq 0$, in particular for

$$
\frac{\lambda}{\gamma} \leq-\int_{0}^{1} \frac{1-x}{\sqrt{x-\log x-1}} \sim-1.55061
$$

no value of $K$ can satisfy the normalization condition (10). This fixes a critical value for our parameter $b$ : if $b<b_{\text {min }}$ the sistem is unstable. Therefore the solution of Haissinski does not exist and the mapping gives a chaotic behavior for the second order moments. The value of $b_{\min }$ in the mapping depends on the values of the physical parameters. Increasing the superperiodicity accordingly to (8) the value of $b_{\min }$ shifts.

In order to compare the results obtained from the mapping with $N_{s}>>1$ and those from the Haissinski equation, we consider the longitudinal density

$$
u(x)=\int_{-\infty}^{+\infty} \psi(x, y) d y
$$

and compare that one obtained from the mapping with $N_{s}=150$ and the solution of the Haissinski equation (9). The comparison of the longitudinal densities for different $b$ is shown in Fig 3. We found that approaching the threshold value $b_{\min }$ the comparison becomes more and more meaningless.

\section{CONCLUSIONS}

For $b>0$ and superperiodicity $N_{s}>>1$, the mapping of the second order moments shows stable solutions of period 1 and period $n$, accordingly to what happens with $N_{s}=1$ [4]. For $b<0$ and $N_{s}>>1$ the solution is stable only in a

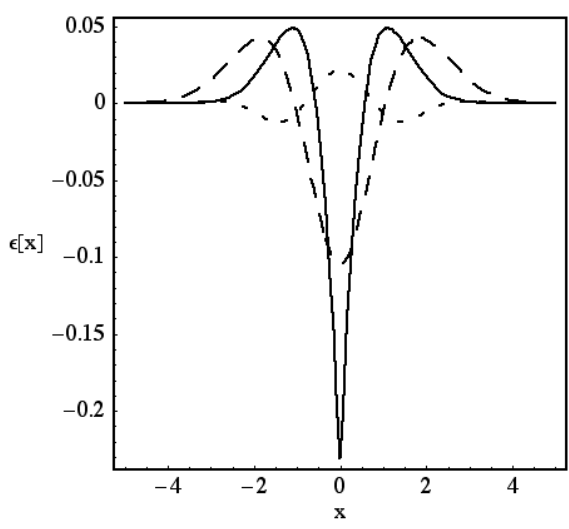

Figure 3: The comparison of the longitudinal densities $u_{H}(x)-u_{m}(x)$ for different $b: b=0.4$ (dotted line), $b=-0.1$ (dashed line), $b=-0.416$ (solid line). $u_{m}(x)$ is computed from the mapping with $N_{s}=150$ and $u_{H}(x)$ numerically from the PWD equation .

small interval of $b$ values: below a critical value of $b$ the solution of the mapping is unstable and furthermore a chaotic behavior appear; corrispondingly the Haissinski equation does not have solution. This result is obtained by numerical iteration of the mapping and it should be discussed more carefully.

While the Haissinski equation can give stable solutions and predict only the nonexistence of stable solutions, the mapping (the Gaussian approximation) shows also what kind of instability (successive period-doubling bifurcations, coexistence of multi-periodic states or chaos) can occur in the parameter space for the bunch length and the energy spread.

As further application of the Gaussian approximation we plan to consider a regularized inductive wake function (which restores the existence of solutions of Haissinski equation below threshold) as recently discussed in [6].

Moreover we will compare our mapping results with those obtained from MPT, as done in [1] and [7], where in the simplest case of constant wake function a good agreement with MPT was found.

\section{REFERENCES}

[1] K. Hirata, S.Petracca and F. Ruggiero, Phys. Rev. Lett., 66, N. 13, 1991, 1693

[2] A.W. Chao, Physics of Collective Beam Instabilities in High Energy Accelerators, J. Wiley \& Sons, 1993.

[3] K. Hirata and F. Ruggiero, Part. Acc., 28, 1990, 137 and LEP Note 611 (1988).

[4] K. Hirata, E.S. Kim, KEK Report 95-5 (1995).

[5] S. Petracca, Part. Acc. 1993, vol. 42(1), p. 45-64.

[6] Y. Shobuda, K. Hirata, KEK Preprint 99-37, July 1999, A.

[7] K. Hirata, Part. Acc. 1987, vol. 22, p. 57-59. 